\title{
Los desafíos de la nueva geografía política en Colombia
}

\section{Miguel Borja*}

Resumen. El artículo presenta los desafíos que la nueva geografía polí tica tiene en Colombia. Se hace un llamado de atención a nuestras clases dirigentes y a la ciudadanía para que otorguen la importancia debida a la geografía política en las tareas de delimitación de las fronteras, las áreas marítimas y la órbita geoestacionaria. Igualmente, se esbozan las labores del momento para los geógrafos políticos: la implementación de los manda tos constitucionales en torno al ordenamiento territorial, la formulación de soluciones para detener la pérdida de control territorial por parte del Estado y la implementación de una geografía de la diversidad y de la globalización. Palabras clave: Geografía política, ordenamiento territorial, entidades territoriales, conflicto armado, control estatal del territorio y geopolítica global.

* Sociólogo de la Universidad Nacional. Magister en Estudios Políticos del IEPRI. Profesor Asistente de la Escuela Superior de Administración Pública. Catedrático del Programa de Estudios de Posgrado en Geografía. Convenio UPTC-IGAC. Catedrático de la Universidad Nacional de Colombia. 


\section{A manera de introducción}

Cada vez resulta de mayor claridad que el Estado moderno tiene como una de sus tareas centrales la de responder por la administración y gestión pública de los territorios que conforman la nación y posibilitan la soberanía. Estas labores constituyen en su generalidad el terreno de estudio propio de la geografía política, la cual como una rama bifronte entre la geografía y las ciencias políticas busca determinar las relaciones que se dan entre el Estado y su territorio, entre el mundo de la política y el espacio, entre las formas que el poder adquiere socialmente y su expresión geográfica.

Actualmente, la nueva geografía política realiza una serie de esfuerzos encaminados a trazar saberes que posibiliten una praxis sobre el territorio, con el fin de colaborar en los esfuerzos de cambio en asuntos como el ordenamiento y la organización territorial de los estados y las sociedades.

Reformas encaminadas a instaurar la estatalidad de los contenedores territoriales que subyacen bajo el manto veleidoso de los Estados nacionales que la Sociedad de Occidente a partir del autoritarismo político y la ficción jurídica consagró como englobadores políticos. La mirada de la nueva geografía política está dirigida a las formaciones regionales, provinciales o comarcales y a los espacios que constituyen o pueden dar lugar a la conformación de las geografías del lugar.
Los debates en el terreno de la geografía política adquieren particular relevancia en un país que como Colombia se empeñó desde los años noventa en la realización de reformas territoriales que modifiquen la estructura de la geografía político-administrativa. A partir de la Constitución de 1991, temas como el del ordenamiento territorial y la geopolítica interna, comenzaron a ser debatidos por diferentes actores sociales. Entre ellos se pueden mencionar, los movimientos regionales y locales, y algunos pocos miembros de los partidos políticos y las universidades, preocupados por el tema.

De esta forma, comenzó a surgir una comunidad interesada en encontrar respuestas al desafío de organizar el territorio nacional y sus regiones, con el fin de atender las demandas sociales para el manejo de la geopolítica, la biodiversidad, las culturalidades, el mar y sus costas y la órbita geoestacionaria. Esta comunidad ha puesto de presente la necesidad de trazar nuevas políticas y programas para gestionar el espacio público, dinamizando así la discusión sobre la geografía política.

Lo anterior se ha dado a pesar del desdén tradicional que el Estado, la universidad y las clases dirigentes han exhibido en la historia del país. Desinterés que ha coadyuvado a la desorganización en la administración y gestión pública del espacio nacional, lo cual ha dado lugar al nacimiento de una serie de geografías paralelas al mapa oficial de la República, funcionales a los actores de la guerra, los desequilibrios regionales y las tensiones 
geopolíticas. Por esto, el artículo busca llamar la atención del Estado y la sociedad colombiana sobre la importancia de los aportes que la geografía política puede hacer para ayudar a resolver los asuntos relacionados con la administración pública del territorio, en el camino hacia la construcción de mejores modelos estatales para la gestión de la geografía colombiana.

\section{Consecuencias del desconoci- miento de la geografía política por parte de los funcionarios públicos}

Ante las dificultades surgidas para el buen gobierno de la geografía nacional el Estado y la sociedad, no pueden continuar con la actitud tradicional de nuestros estadistas y gobernantes de dejar a un lado los asuntos relacionados con la geografía política. Por cuanto la geografía política como un campo de las ciencias sociales que busca dilucidar las relaciones entre los fenómenos políticos y estatales y el espacio geográfico, no sólo adquiere cada vez una mayor importancia en la trama del Estado contemporáneo, sino que nos permite comprender los procesos históricos a través de los cuales las comunidades se organizan políticamente bajo formas estatales y determinan un conjunto de geografías político-administrativas.

Recordemos que las comunidades al construir socialmente las territorialidades terminan por ordenar sus áreas geográficas para una mejor gestión de los asuntos sociales y políticos. Este orden territorial da nacimiento a una serie de programas e instituciones dedicadas a organizar la administración y gestión territorial del Estado, campo de investigación y acción participativa que constituye el terreno privilegiado de la geografía política, haciendo de ella uno de los elementos básicos para la inserción de las comunidades nacionales y regionales en la comunidad global y la definición de unos mínimos niveles de gobernabilidad que posibiliten la convivencia pacífica y el despliegue económico de la sociedad.

Por consiguiente, no tener en cuenta los aportes científicos y técnicos que brinda la geografía política para el manejo de los asuntos territoriales del Estado, no solamente posibilita la desorganización territorial, sino también la fragmentación y desmembración del espacio nacional.

Ante las dificultades surgidas para el buen gobierno de la geografía nacional el Estado y la sociedad, no pueden continuar con la actitud tradicional de nuestros funcionarios públicos de dejar a un lado los asuntos relacionados con la geografía política. Pues esta actitud, no solamente posibilita la desorganización territorial, sino también la fragmentación y desmembración del territorio.

Dicha línea de conducta para el manejo de los asuntos públicos, fue la que permitió que durante la delimitación de las fronteras internacionales se perdiera gran parte del territorio inicial que le correspondía a la Nueva Granada, de acuerdo con el principio del Utis Possidetis Juris de 1810. La desafortunada actuación de la administración pública en los procesos de delimitación de las fronteras, llevó a la 
pérdida de áreas geográficas, que de acuerdo con el derecho internacional le correspondían a Colombia. Asimismo, el país se vio obligado a enfrentar conflictos armados con el Perú, Ecuador, Panamá y Nicaragua, motivados por los proyectos expansionistas de dichas naciones. Esta tragicomedia del Estado dejó a las fronteras internacionales como una línea de tensión territorial, en donde permanentemente la soberanía es puesta en duda.

Obsérvese lo que sucede con nuestro mar territorial frente al Golfo de Coquibacoa. Después de ceder diversas franjas territoriales en los acuerdos de delimitación con Venezuela, que llevó incluso a que los negociadores colombianos admitieran la noción de costa seca y a que Venezuela apareciera con una franja de territorio en la Guajira, mediante una nota diplomática del 22 de noviembre de 1952, se cedieron los Monjes a Venezuela. La entrega de los Monjes ha influido notablemente en la delimitación de las áreas marinas en el Golfo, permitiéndole al vecino país, no solo dilatar las conversaciones, sino también aumentar sus aspiraciones y realizar ejercicios de soberanía en áreas marinas aún no demarcadas.

El desmembramiento del territorio es resultado del hecho de que el Estado y sus funcionarios han descuidado los asuntos relacionados con la geografía política. Este desinterés conduce a que los encargados de las tareas geopolíticas no tengan ni hayan generado un sentido geográfico en su actuación profesional. Por consiguiente, los gobiernos no han animado sus relaciones internacionales de acuerdo con un imaginario geográfico que proyecte a la comunidad política, en el marco de las naciones.

\section{Rol de la geografía política en la inserción internacional}

En vía de ilustración se puede señalar que, debido a la ausencia del vector de la geografía política y social en la formación de nuestros gobernantes, aún no se ha podido jugar el papel que nos corresponde en América Latina en el campo de la definición de las relaciones internacionales y de la geografía política regional. Los asuntos relacionados con las negociaciones en el plano internacional, han quedado casi siempre en manos de abogados, quienes no conocen los elementos mínimos de geografía necesarios para tener un rol decoroso en la escena regional. Es por esto, que los negociadores, llegado el momento de definir los asuntos relacionados con la demarcación de los límites terrestres y marítimos y la órbita geoestacionaria, generalmente se encuentran mal informados y precariamente preparados, situación que los lleva a ceder áreas estratégicas del espacio colombiano.

La despreocupación geográfica de las elites dirigentes, no puede continuar haciendo carrera en las organizaciones e instituciones estatales. Pues uno de los elementos centrales del poder estatal lo constituye la geografía política, la cual determina la soberanía y es la base territorial necesaria para la edificación de ciudadanías y de esferas públicas. 


\section{La geografía política en el debate público}

La formación de nuestros ciudadanos en general, y en particular de los gobernantes y funcionarios públicos, en el campo de la geografía política, es aún más urgente si se tiene en cuenta que desde los años noventa la reflexión sobre la administración pública del territorio, ocupa un lugar destacado en la agenda de los gobiernos locales y regionales y de los partidos políticos, los gremios económicos y diversos movimientos sociales y culturales.

En los debates sobre la administración pública del territorio se ha mostrado que el país no cuenta con una geografía política adecuada para el manejo de los asuntos económicos y sociales, hecho que ha llevado al diseño y puesta en marcha de una serie de políticas encaminadas a reformar la gestión del territorio. Acciones que se concretaron parcialmente, mediante la expedición de la Constitución de 1991, la ley de fronteras, de reforma territorial y de negritudes. Esta anterior normatividad es básica para el rediseño de las políticas estatales para la administración del territorio.

En este nuevo derecho territorial se contempla la posibilidad de establecer regiones y provincias, la flexibilidad de los límites internos y el manejo especial de territorialidades no convencionales como los territorios indígenas, los territorios ribereños de las comunidades negras y las zonas de frontera. Elementos que conforman un punto de partida para superar el desorden en la dirección política de los territorios, pero que no han sido implementados por los gobiernos posconstituyentes.

La desidia pública ha llevado a que la geografía política continúe desconociendo los procesos de readecuación espacial entre el campo y la ciudad, los fenómenos de conurbación, las territorialidades culturales, los territorios de la colonización, las zonas de frontera, el rápido crecimiento demográfico $\mathrm{e}$ industrial, la ampliación de la frontera ganadera y agrícola, y las dinámicas de las fronteras internas y externas. Además, se ha podido comprobar que la acción deficiente del Estado en el terreno de la geografía política, dificulta el manejo eficiente del medio ambiente, las finanzas públicas, la vida política y electoral, el desarrollo regional y la inserción de la nación como parte codeterminante de la sociedad global.

\section{4. ¿Quién debe dar respuesta a las anteriores falencias?}

Frente a esta situación las instituciones encargadas de la geografía política, entre ellas la universidad, no pueden permanecer indiferentes. Deben en primer lugar entrar a traducir en políticas públicas y en programas políticos $\mathrm{y}$ sociales, el nuevo derecho territorial. Máxime aún cuando las entidades territoriales de la nación, los actuales departamentos y municipios, vienen enfrentando una serie de dificultades en 
sus finanzas públicas y en los niveles de gobernabilidad.

Igualmente, la intelligentsia en un espacio nacional signado por la guerra civil se debe entrar a plantear soluciones para dar respuesta a la pérdida de control territorial por parte del Estado, la cual tiene diversas expresiones. En primer lugar, un dominio estable de las organizaciones armadas sobre algunas zonas geográficas y la población allí asentada. En segunda instancia, el control relativamente estable del acceso a regiones y corredores geoestratégicos. Y finalmente, el control temporal de vías de comunicación vitales para el país, hecho que produce un impacto político directo sobre la opinión nacional e internacional.

La sociedad y el Estado, esperan que sus geógrafos políticos planteen políticas y acciones gubernamentales encaminadas a la superación pacífica del conflicto armado en general, y en particular a diseñar estrategias encaminadas a recuperar el dominio del territorio para el Estado y las comunidades. Asimismo, se espera que los geógrafos políticos estén en capacidad de gestionar el posdesarrollo regional en las territorialidades culturales. En este sentido, los Territorios Indígenas, los Territorios Ribereños de las Comunidades Negras, las Zonas de Frontera, las Zonas de Colonización son un desafío para la geografía política. Campo del saber que deberá abandonar las coordenadas tradicionales del Estado-nación, para dar respuesta a las particularidades que implica la gestión territorial de la diversidad geográfica.

Es urgente llamar la atención de la clase política y las universidades, para que entren a preparar planes y programas, que más adelante se traduzcan en políticas públicas, para el manejo de la diversidad territorial de lo público, que se expresa en términos geográficos en la necesidad de manejar las territorialidades regionales.

\section{5. ¿Es acertado el rol actual de los departamentos y municipios para el trazado de geografías políticas coherentes?}

Las lecciones recientes de la tragedia nacional, muestran que la geografía política no puede seguir teniendo como horizonte a los departamentos y municipios, entidades territoriales que posiblemente cada vez jueguen un papel menor en la estructura de la administración pública.

El departamento, instaurado para imponer a través del autoritarismo estatal unas instituciones políticas extrañas al espíritu de las diferentes formaciones socioeconómicas que existen en el país, ha tenido una vida institucional errática y es una de las instancias generadoras de las diversas crisis que ha atravesado la historia de la nación durante los últimos cien años. Trazados sus límites de manera arbitraria y en muchas ocasiones con el fin de responder a las aspiraciones burocráticas y políticas del bipartidismo, se ha convertido en uno de los elementos 
fundamentales de la desorganización territorial. Las administraciones departamentales son un agregado extraño a la vida de las comunidades. Las autoridades regionales a pesar de su elección popular, continúan siendo figuras alejadas de los espacios territoriales donde ejercen su mandato. Pues, por ejemplo, los gobernadores son elegidos ante todo por los pobladores de las ciudades en las cuales se concentra la mayor parte del censo electoral. Por tanto, los municipios medianos y pequeños y las áreas rurales continúan sin una vida política autónoma y activa.

Súmese a lo anterior el hecho de que, los departamentos presentan numerosas disfuncionalidades ocasionadas por el deficiente trazado de sus límites, las cuales van desde las ecológicas hasta las de orden público. Igualmente, ellos concurren al desorden en la administración pública de los asuntos territoriales. Por ejemplo, tres departamentos y 14 municipios se disputan la administración de la Sierra Nevada de Santa Marta. Siete departamentos concurren en la administración de una región de alto conflicto social como el Magdalena Medio. Adiciónese a lo anterior que las administraciones seccionales son feudos histórico-políticos. En ellas, lo dominante es el prebendalismo estatal y la aeticidad generalizada de sus empleados y funcionarios. Los recursos públicos desaparecen por artes mágicas y profanas. Existe un consenso que muestra a los departamentos como los principales focos del clientelismo, la burocratización, el despilfarro financiero y la ineficiencia administrativa.
Algo similar pasa con los municipios. Pues ellos, al igual que los departamentos no son espacios con identidad territorial de acuerdo con las características naturales, sociales, económicas y culturales, que la geografía política moderna utiliza para delimitar los territorios. Asimismo, gran parte de los municipios fueron creados como entes para la feudalización del poder político. El desorden territorial clásico de nuestras instituciones viene haciendo del municipio una estructura que no posibilita la implementación de políticas como la descentralización, la reforma política, la democracia local ni la modernización del Estado. Basta con saber, que al igual que los departamentos, la mayor parte de los municipios no son financieramente viables.

En consecuencia, ni el departamento ni el municipio son plataformas territoriales para la implementación de una administración pública moderna.

\section{La Constitución de 1991 y la geografía política}

Afortunadamente, la Constitución de 1991 estableció la posibilidad de instaurar a las regiones, provincias, áreas metropolitanas, territorios indígenas, territorios de las comunidades negras ribereñas y otras entidades como unidades para la administración territorial, quienes pueden ser una respuesta adecuada al desorden político-administrativo. La geografía política está llamada, entonces, a diseñar formas y modelos administrativos que superen la tríada na- 
ción, departamento y municipio, y que permitan interpretar los cambios legales que se vienen dando.

Agréguese a lo anterior la urgencia que existe para que los funcionarios públicos interpreten los cambios en la geografía política que introdujo la Constitución a partir de los principios de flexibilidad de la geopolítica interna, equilibrio y solidaridad regional, autonomía territorial y rotación geográfica del poder. La flexibilidad de la geografía del Estado puede ser considerada desde dos ángulos. En primer lugar, el artículo 290 de la Constitución nacional, estableció la realización del examen periódico de los límites de las entidades territoriales, el cual es un punto de arranque para la formulación y la puesta en marcha de una nueva administración del territorio a partir de la revisión y la adecuación de los límites internos.

Desde otra perspectiva, la Asamblea Nacional Constituyente dio vía libre a la superación de la tríada geopolítica conformada por la nación, los departamentos y los municipios, la cual es considerada obsoleta para el manejo del país y sus regiones. La Asamblea abrió la posibilidad de regionalizar y subregionalizar el territorio, con el fin de establecer una cartografía adecuada para el manejo de las geografías públicas.

Igualmente, el principio de la autonomía territorial es un punto de partida para la construcción de las autonomías políticas. La autonomía es la matriz generadora de geografías políticas que posibiliten la organización del territorio con el fin de establecer niveles mínimos de gobernabilidad y desarrollo social. El derecho de las entidades territoriales a gobernarse por autoridades propias, elegidas democráticamente, el derecho de cada entidad territorial a ejercer libremente las competencias constitucionales y legales que le correspondan; el derecho de cada entidad a administrar sus recursos propios y a establecer los tributos necesarios para el cumplimiento de sus funciones; y el derecho a participar en las rentas nacionales, tal y como lo señala la Constitución, son los fundamentos legales para hacer de la autonomía territorial la estrella polar para el ordenamiento territorial de la nación.

De este modo será posible reordenar el territorio a partir de coordenadas que interpreten la transformación de los límites de las unidades espaciales de acuerdo con la apropiación y el uso del territorio por parte de las sociedades comunitarias. Asimismo, se posibilitará el cambio en los actuales límites internos, que no corresponden al funcionamiento real de las regiones y son uno de los principales obstáculos para la gestión y la gobernabilidad.

\section{Las tareas de la nueva geografía política en la sociedad global}

Pero si, las nombradas labores son algunas de las tareas coyunturales de la geografía política, existen trabajos de mayor alcance puesto que la gestión del territorio, además del orden territorial interno, implica un conjunto de variables interna- 
cionales. En la ciencia política tradicional el territorio de un Estado se delimita por unas fronteras, y a partir de ellas se comienza a señalar su papel en el contexto mundial. Pero a pesar de esta tradición, en la sociedad global las territorialidades ya no pueden ser construidas como nichos geográficos, la economía de geografía variable derrumba fronteras y murallas chinas, compone y recompone la cartografía mundial y las soberanías políticas.

Surge una nueva geografía política, en la cual se dan procesos de defragmentación y fragmentación territorial, de apertura de fronteras y de creación de nuevos imaginarios geográficos. Se construye una nueva praxis geopolítica en la que vuelve a resurgir el interés por los problemas del espacio. Interés ilustrado en la actualidad por la importancia que adquiere la reflexión sobre las relaciones entre la globalización y la geografía política.

Este volver sobre el tema del espacio transforma las prácticas y las representaciones colectivas como las vinculadas con la noción de estado territorial. Cada vez se desdobla más la imagen territorial del espacio mundial como un mundo dividido en diferentes unidades nacionales. La nueva praxis geopolítica en la sociedad global determina diversas territorialidades, construyendo espacios favorables para el despliegue de las eco- nomías globales y dejando por fuera amplias áreas como territorios marginales. Asimismo, los procesos de internacionalización determinan el surgimiento de acuerdos y formas de representación política, estimulando la formación de hegemonías encaminadas a establecer modelos de desarrollo y reglas de juego favorables para los actores globales. Procesos que se dan por fuera de las rígidas estructuras del Estado nacional.

Pero quizás el hecho más importante es que los escenarios internacionales que se están conformando, obligan a pensar toda propuesta de futuro "en función del posicionamiento en los mercados internacionales, en función de un reparto más equitativo de las ganancias, con suficiente flexibilidad para acomodar el territorio en diferentes ámbitos y configuraciones territoriales, administrándolo como si fuese una organización empresarial, gobernándolo de manera de crear poder político, demandando al Estado una visión territorial de sí mismo, y entendiendo que una gestión contemporánea requiere crear "capital socio-geográfico, sin que ella se agote en el uso de los recursos materiales tradicionales". ${ }^{1}$ Las tendencias mundiales obligan a colocar los pilares para la construcción de una praxis geopolítica que permita a nuestros conciudadanos jugar un papel destacado en la inserción en el mundo de la nación, sus regiones y localidades. Posibilitando el diseño de

1 S. Boisier, 'Conocimiento y gestión territorial en la globalización', en Estudios Internacionales, jul-dic, 1997, No. 119-120, Santiago de Chile, Instituto de Estudios Internacionales de la Universidad de Chile, pp. 275-292. 
proyectos de futuro a partir de una administración moderna del territorio.

En consecuencia, en lo que hace relación con la geografía territorial del Estado es necesario empezar a generar geografías políticas que posibiliten a las comunidades y a las instituciones entrar a liderar y orientar los procesos de ordenamiento territorial interno y los caminos para la inserción internacional a partir de los proyectos geoestratégicos y territoriales del Estado.

\section{Conclusiones}

La administración pública del territorio en Colombia se puede considerar una geopolítica inadecuada para la vida social y política. En el campo internacional, el país pasó de tener unas fronteras en relativa calma a unas fronteras de tensión. Los países vecinos se han dedicado a establecer colonizaciones como fronteras de avanzada y a tender un cordón de seguridad a lo largo de los límites biestatales. Igualmente, se ha venido creando un imaginario geopolítico para presentar a la nación como una amenaza para la seguridad regional en América Latina.

$\mathrm{Y}$ si lo mencionado sucede internacionalmente, en el plano interno la administración pública del territorio se caracteriza por el desorden, el cual se manifiesta directamente en la inexistencia de una geografía política coherente para la gestión de lo público.

Problemas como las tensiones geopolíticas internas y externas, los desequilibrios regionales, el desplazamiento interno, los conflictos armados por el control de áreas geográficas, la ingobernabilidad y el mal manejo del medio ambiente y los recursos naturales, tienen como uno de sus elementos generadores la ausencia de una adecuada gestión pública del territorio, que le permita a la nación y sus regiones tener en su geografía política un elemento para la gestión del espacio y para la inserción de la nación en la economía global.

En consecuencia, en lo que hace relación con la geografía territorial del Estado es necesario empezar a generar nuevas geografías que posibiliten a las comunidades y a las instituciones entrar a liderar y orientar los procesos de ordenamiento territorial interno y los caminos para la inserción internacional a partir de los proyectos geoestratégicos y territoriales del Estado. Estas son precisamente tareas de la geografía política en el país, para lo cual es necesario entrar, por un lado, a revalorar sus aportes en la formación de nuestros ciudadanos, políticos y funcionarios públicos $\mathrm{y}$, por otra parte, sus posibles contribuciones en la construcción de órdenes territoriales que permitan superar las carencias del Estado territorial. 